\title{
Massive Colchicine Overdose with Recovery
}

\author{
Ioulia losfina $^{a} \quad$ James Lan $^{\mathrm{b}}$ Carson Chin $^{\mathrm{a}}$ Ronald Werb \\ Adeera Levin ${ }^{\mathrm{b}}$
}

Departments of ${ }^{\mathrm{a}}$ Medicine and ${ }^{\mathrm{b}}$ Nephrology, University of British Columbia, Vancouver, B.C., Canada

\section{Key Words}

Colchicine $\cdot$ Overdose $\cdot$ Dialysis $\cdot$ Sepsis

\begin{abstract}
Colchicine is an active alkaloid that is commonly used for treatment of multiple diseases including gout, primary biliary cirrhosis and familial Mediterranean fever. Less commonly, it has been implicated in several fatal overdoses. Deaths from colchicine overdoses are usually due to multi-organ failure, whether directly from colchicine toxicity or due to ensuing sepsis. We report an extreme case of colchicine ingestion $(1.38 \mathrm{mg} / \mathrm{kg})$, which is the largest reported non-fatal colchicine overdose. The patient was a 47 -year-old First Nations woman with a history of depression and no other comorbidities. Ingestion was intentional and initial presentation was within $2 \mathrm{~h}$ of ingestion, at which point she had normal clinical and laboratory parameters. Early implementation of a targeted therapeutic strategy directed at the predicted multi-organ failure which included aggressive use of a GI decontamination protocol, timely supportive measures including ventilator support and renal replacement therapy, as well as the utilization of broad-spectrum antibiotics and G-CSF for sepsis and leucopenia management, resulted in successful support and discharge of this patient off dialysis.
\end{abstract}

\section{Introduction}

Colchicine is used for the treatment of gout, familial Mediterranean fever, primary biliary cirrhosis, amyloidosis and condyloma acuminate. Despite its merits, its narrow therapeutic index has been the source of several incidences of successful suicides by overdose [1]. Deaths from colchicine overdoses are uncommon but well documented, and are associated with a high mortality rate. We present here a case of a profound colchicine overdose, potentially the largest consumption reported in the literature, from which the patient successfully recovered. A multifaceted, aggressive set of interventions, instituted within $24 \mathrm{~h}$ of ingestion may well have been the reason for the

Dr. Ioulia losfina 
successful outcome for this patient. Appreciating the mechanism of action by which colchicine causes cell damage and instituting supportive therapies are important learnings from this case.

\section{Case Presentation}

A 47-year-old Aboriginal female was admitted to a hospital in rural British Columbia for attempted suicide. She had ingested $90 \mathrm{mg}$ of her husband's colchicine an hour prior to admission. She was not on regular medications and her past medical history was remarkable only for depression.

Coingestions included alcohol, in the form of 6 cans of beer. She presented within one hour of ingestion with mild abdominal pain and normal vital signs. Physical exam was unremarkable. In the emergency room, $250 \mathrm{~g}$ of activated charcoal was administered over the course of $12 \mathrm{~h}$. Within the initial presentation, she became febrile, developed diarrhea and severe nausea and vomiting, and developed refractory acidosis requiring urgent transfer to a tertiary hospital for further management $24 \mathrm{~h}$ post ingestion (fig. 1 ).

At transfer, her vitals were: BP 99/61 mm Hg, HR 106 bpm, RR 26 bpm and T 37.4 ${ }^{\circ} \mathrm{C}$. She had bibasilar inspiratory crackles and chest X-ray showing bilateral infiltrates at the bases. Sodium bicarbonate infusion and fluids were started to treat her acidosis. Over the next $24 \mathrm{~h}$ she received $50 \mathrm{~g}$ of activated charcoal every $6 \mathrm{~h}$. She was also given N-acetylcysteine (NAC) loading dose of $150 \mathrm{mg} / \mathrm{kg}$ IV over $1 \mathrm{~h}$ followed by $50 \mathrm{mg} / \mathrm{kg}$ IV over $4 \mathrm{~h}$ then $100 \mathrm{mg} / \mathrm{kg}$ IV over the next $16 \mathrm{~h}$.

Despite the resuscitative and treatment measures described above, she developed multi-organ failure $48 \mathrm{~h}$ after ingestion (table 1). She had evidence of cardiovascular collapse with myocardial damage, renal failure and liver failure and altered level of consciousness, with a normal head CT. Hemodialysis was started for acute oliguric renal failure and refractory acidosis. At $72 \mathrm{~h}$ post ingestion she became febrile and pancytopenic, and required supportive transfusions. Febrile neutropenia protocol was initiated when WBC fell below $1.0 \times 10^{9} / \mathrm{l}$ : this included broad spectrum antibiotics and G-CSF. Blood cultures were positive for Moraxella and sputum was positive for Klebsiella. At 5 days post ingestion, she developed septic shock and required intubation. She also developed rhabdomyolysis and was treated supportively with fluids and continued dialysis.

She was extubated 2 weeks post ingestion after resolution of her sepsis but continued to receive intermittent hemodialysis. Liver function also gradually improved. The patient continued to suffer from alopecia throughout the admission, and complained of hearing loss in her left ear that resolved spontaneously. She also developed gangrene in her toes likely secondary to septic shock and vasopressor use. One month after ingestion, she regained normal urine output and kidney function, and intermittent hemodialysis was discontinued. She was discharged home in stable condition with scheduled follow-up.

\section{Discussion}

Colchicine toxicity occurs through the interruption of mitosis by preventing polymerization of tubulin into microtubules. Therefore, although colchicine is absorbed in all cells of the body, it most adversely affects cells with increased mitotic activity such as those in the GI tract and in the bone marrow [1]. Colchicine poisoning classically presents in three stages. The initial stage at $<24 \mathrm{~h}$ is characterized by GI symptoms such as abdominal pain, nausea, vomiting and diarrhea. The second phase from day 2 to 7 post ingestion is the most dangerous: it is characterized by multi-organ failure, involving bone marrow suppression, kidney and liver failure, ARDS, arrhythmias and cardiovascular collapse, and neuromuscular involvement. The third stage is only seen in patients who recover from colchicine poisoning. It usually starts 
after day 7 when there is resolution of organ failure, rebound leukocytosis and alopecia [2].

The pharmacokinetics of colchicine are complex. It is rapidly absorbed from the GI tract and is metabolized primarily by the liver, which involves de-acetylation following first-order kinetics. Subsequently, there is significant entero-hepatic recirculation with $10-20 \%$ renal excretion [2]. The drug's clearance is significantly prolonged in patients with hepatic and renal insufficiency. After absorption, colchicine is rapidly distributed to all tissues where it is heavily protein bound.

Filkenstein et al. [1] have reported high fatalities after ingestion of $>0.5 \mathrm{mg} / \mathrm{kg}$ of colchicine, with the lowest reported lethal doses ranging from 7-26 mg. In a large French series examining colchicine overdoses, doses of $>0.8 \mathrm{mg} / \mathrm{kg}$ almost always caused death within $72 \mathrm{~h}$ [3]. Our patient ingested approximately $1.38 \mathrm{mg} / \mathrm{kg}$ of colchicine, which is the highest non-fatal dose of colchicine that has been reported. Her survival is likely due to a combination of early presentation, timely intervention with activated charcoal, NAC and precise supportive therapy for multi-organ failure.

Our patient was fortunate to present within one hour of ingestion. Activated charcoal was started within $2 \mathrm{~h}$ of ingestion and was continued for a total of $36 \mathrm{~h}$. The prolonged use of activated charcoal was aimed to target the extensive entero-hepatic recirculation of colchicine. Due to the high affinity of colchicine to plasma proteins and its large volume of distribution, both hemodialysis and hemoperfusion play a limited role in the acute management of colchicine toxicity. Interestingly, colchicine-specific Fab fragment antibodies have been used successfully in the treatment of severe colchicine intoxication; however, such treatment modality is not commercially available in Canada and precluded its use in our patient [1].

Significant amounts of NAC were also used in this patient. We utilized the NAC protocol for acetaminophen overdose, as no specific NAC protocol is available for colchicine. NAC has previously been used in the treatment of a variety of conditions including acetaminophen overdose, HIV/AIDS, cystic fibrosis, etc. It exhibits anti-oxidant properties by reducing oxidant-induced cell damage and cell death by apoptosis [4]. We hypothesized that NAC used in this patient may have counteracted the inhibiting effects of colchicine on the endogenous anti-oxidants and may have decreased cell death by apoptosis and contributed to her survival despite the extremely high dose of colchicine ingested.

The exact mechanism of colchicine toxicity on kidney failure is not known. It may be due to multiple factors such as hypotension, volume depletion, rhabdomyolysis and multi-organ failure. However, it is possible that colchicine may have a direct toxicity on the proximal renal tubules. Cases of severe intoxication invariably demanded renal replacement therapy as a supportive measure for metabolic acidosis, progressive anuria and uremia [5]. Our patient fortunately recovered her kidney function 5 weeks post ingestion, demonstrating the reversibility of acute kidney injury due to colchicine. As a biopsy was not performed, the exact areas of damage cannot be determined.

In addition to the standard supportive therapies for multi-organ failure, special attention was given to the management of sepsis. Both fever and an elevated WBC may be unreliable indicators of sepsis in the face of colchicine intoxication. Persistent fever is often a feature of colchicine toxicity itself in the absence of sepsis; as for peripheral 
WBC, it begins with peripheral leukocytosis following acute ingestion, which is then followed by leucopenia in stage 2 of intoxication; finally, one observes rebound leukocytosis in the resolution phase [2]. In our patient, febrile neutropenia protocol proved to be life-saving as she proved to have both bacteremia and pneumonia.

However, in the resolution phase her antibiotics were judiciously stopped as her fever and leukocytosis then were more consistent with the rebound effect of colchicine toxicity rather than with sepsis.

By the end of her hospital stay, our patient's main symptoms were alopecia subtotalis, and gangrene in her toes, which was likely attributed to an aggressive use of inotropes and pressors in the face of cardiovascular collapse.

In summary, we present a case that demonstrates successful recovery after a massive colchicine overdose, despite complications of multi-organ failure and prolonged dialysis. We demonstrate that understanding the physiologic toxic effects of colchicine and addressing them in a multi-interventional approach may prevent future mortalities from overdose of this drug.

Table 1. Multi-organ failure: lab values for the first week of admission

\begin{tabular}{lllllllllll}
\hline & $1 \mathrm{~h}$ & $12 \mathrm{~h}$ & $18 \mathrm{~h}$ & $24 \mathrm{~h}$ & $48 \mathrm{~h}$ & $72 \mathrm{~h}$ & $96 \mathrm{~h}$ & Day 5 & Day 6 & Day 7 \\
\hline WBC, $10^{9} / \mathrm{l}$ & 5.2 & 17.5 & 25.9 & 23 & 8.9 & 3 & 1 & 1.1 & 9.1 & 29.4 \\
Cr, $\mu$ mol/l & 52 & 58 & 65 & 80 & 205 & 207 & 284 & 215 & 161 & 143 \\
AST, U/l & 29 & 96 & 218 & 364 & 12,618 & 15,111 & 9,830 & 3,938 & 1,172 & \\
Troponin, $\mu \mathrm{g} / \mathrm{l}$ & & & $<0.01$ & 0.31 & 7.99 & 4.7 & & 0.79 & & 0.24 \\
CK, U/l & & \multirow{2}{*}{121} & 358 & & 3,035 & & & 28,064 & 8,194 & 1,862 \\
\hline
\end{tabular}



Fig. 1. Case timeline and management. 


\section{References}

1 Filkenstein Y, Aks SE, Hutson JR, Juurlink DN, Nguyen P, Dubnov-Raz G, Pollak U, Koren G, Bentur Y: Colchicine poisoning: the dark side of an ancient drug. Clin Toxicol 2010;48:407-414.

2 Maxwell MJ, Muthu P, Pritty PE: Accidental colchicine overdose: a case report and literature review. Emerg Med J 2002;19:265-267.

3 Bismuth $\mathrm{C}$, Gaultier M, Conso F: Aplasie médullaire après intoxication aiguë à la colchicine. Nouv Presse Med 1977;6:1625-1629.

-4 Atkuri K, Mantovani JJ, Herzenberg LA, Herzenbert LA: N-Acetylcysteine - a safe antidode for cysteine/glutathione deficiency. Curr Opin Pharmacol 2007; 7:355-359.

-5 Huang WH, Hsu CW, Yu CC: Colchicine overdose-induced renal failure and electrolyte imbalance. Ren Fail 2007;29:367-370. 